\title{
LICENCIATURA EN EDUCACIÓN FÍSICA Nuevo currículo para el 2001
}

\author{
Responsables:* Mariela Herrera \\ Judith Jaramillo \\ Claudia Mallarino
}

El cambio de currículo a la Licenciatura en Educación Física, nace del reto de asignarle identidad a la Educación Física como disciplina, desde el ámbito epistemológico, para enfrentar los desafíos que el nuevo milenio impone a la sociedad. La reconstrucción orgánica de la U.P.N., en cinco Facultades, dos de ellas nuevas, Educación Física y Artes, condujo a que como parte de la reestructuración en la primera se establecieran tres licenciaturas: Educación Física, Deporte y Recreación, como propuestas inteligentes y creativas para involucrar a la Universidad en las soluciones a las diversas problemáticas del país.

La Licenciatura en Educación Física se dispone entonces, a estrenar currículo el primer semestre del año 2001, en tanto que los currículos de las Licenciaturas en Deporte y Recreación se encuentran en proceso de estudio para acreditación.

\section{Objeto de estudio:}

- La experiencia corporal

\section{Objetivos del Programa:}

- Redimensíonar la concepción de Educación Física con base en fundamentos científicos para transformar el imaginario social vigente y las praxis del futuro maestro.

- Contribuir al desarrollo del proyecto pedagógico de la educación nacional y de la UPN.

- Gestar procesos de construcción disciplinar del área.

- Formar un maestro de Educación Física con características de científico social del cuerpo.

- Formar un maestro de Educación Física capaz de interpretar conceptual, legal y académicamente, las modalidades educativas y sus niveles para tomar decisiones de desempeño profesional y diseño curricular.

\section{Campos de acción:}

- Diseñar e implementar currículos especializados y fundamentados científica y técnicamente que respondan al proyecto pedagógico político nacional ideado y los propósitos educativos institucionales.

- Aportar al proceso de construcción teórica de la disciplina, Educación Física, a través de procesos científico-investigativos.

- Tomar decisiones de desempeño profesional en cualquiera de las modalidades y niveles del sistema educativo con base en análisis conceptuales, legales y académicos.

\footnotetext{
${ }^{*}$ Profesoras Facultad de Educación Física, U.P.N. 
- Artícular; interdisciplinariamente, los distintos campos del saber desde la perspectiva de la Educación Física.

- Discriminar características de la actividad física según condiciones y necesidades del grupo objetivo.

- Ejercer prácticas pedagógicas innovativas en las diferentes modalidades y niveles del sistema educativo, que transformen el imaginario social vigente de la Educación Física.

- Elaborar textos y materiales didácticos de calidad académica que ameriten su publicación y divulgación.

\section{Plan de estudios}

\section{Estructura}

Este Currículo se concibe como un sistema complejo, abierto y vivo integrado por tres momentos: Macroestructura que organiza el plan de estudio en ciclos, niveles y áreas, y define los pro pósitos de éstos. Microestructura, que se enmarca en la macroestructura y define los ejes de análisis para las áreas, los núcleos temáticos para las áreas por ciclo y nivel y los temas envolventes (líneas de profundización) para los ciclos y niveles; y el Microdiseño, que es la puesta en acción e implementación de las dos anteriores y define los núcleos proble'micos por ciclo y nivel, y los subproblemas particulares de cada área para el Ciclo I y el Nivel I del Ciclo II. El Nivel IL del Ciclo II, no define subproblemas para las áreas, porque se espera que sean formulados por los estudiantes, como fundamento de su diseño curricular particular. 


\section{MACROESTRUCTURA}

\begin{tabular}{|c|c|c|c|}
\hline & HUMANÍSTICA & PEDAGÓGICA & DISCIPLINAR \\
\hline Áreas & $\begin{array}{l}\text { Propósito: } \\
\text { Aproximación al } \\
\text { conocimiento del } \\
\text { hombre como sujeto } \\
\text { activo en el proceso } \\
\text { de educación formal, } \\
\text { no informal e } \\
\text { informal }\end{array}$ & $\begin{array}{l}\text { Propósito: } \\
\text { Conocer y analizar } \\
\text { críticamente teorías } \\
\text { educativas. }\end{array}$ & $\begin{array}{l}\text { Propósito: } \\
\text { Estudio crítico } \\
\text { de los ejes de } \\
\text { análisis para } \\
\text { construir } \\
\text { conocimiento } \\
\text { específico }\end{array}$ \\
\hline I FUNDAMENTACIÓN & & & \\
\hline $\begin{array}{lr}\text { Propósito: } & \\
\text { Aproximación al análisis y } \\
\text { comprensión } & \text { de } \\
\text { universales } & \text { que } \\
\text { fundamentan } & \text { la } \\
\text { construcción } & \text { del } \\
\text { conocimiento } & \end{array}$ & & & \\
\hline II. PROFUNDIZACIÓN & & & \\
\hline $\begin{array}{l}\text { Nivel 1. Construcción } \\
\text { disciplinar. } \\
\text { Propósito: } \\
\text { Hacer una aplicación } \\
\text { crítica de principios } \\
\text { universales tal que sirva } \\
\text { de herramienta de } \\
\text { análisis y construcción } \\
\text { del saber disciplinar de la } \\
\text { E.F. } \\
\text { Nivel 2. Proyección } \\
\text { Propósito: } \\
\text { Dialogar, debatir y } \\
\text { difundir, sometiendo a } \\
\text { juicio crítico de la } \\
\text { comunidad en general la } \\
\text { propuesta humanístico- } \\
\text { pedagógico-disciplinar } \\
\text { diseñada por el futuro } \\
\text { profesional de la E.F. } \\
\text { como consecuencia de la } \\
\text { implementación de del } \\
\text { currículo aplicado en su } \\
\text { proceso de formación } \\
\text { profesional. }\end{array}$ & & & \\
\hline
\end{tabular}


MICROESTRUCTURA

\begin{tabular}{|c|c|c|c|}
\hline AREAS & HUMANÍSTICA & PEDAGÓGICA & DISCIPLINAR \\
\hline $\begin{array}{l}\text { Ejes de } \\
\text { análisis }\end{array}$ & $\begin{array}{l}\text { Hombre, cultura } \\
\text { y sociedad }\end{array}$ & $\begin{array}{l}\text { Conocimiento, } \\
\text { relaciones de } \\
\text { poder y } \\
\text { comunicación }\end{array}$ & $\begin{array}{l}\text { Experiencias } \\
\text { corporales y } \\
\text { procesos del } \\
\text { desarrollo del } \\
\text { potencial humano }\end{array}$ \\
\hline \multirow[t]{3}{*}{ FUNDAMENTACIÓN } & \multicolumn{3}{|c|}{ Tema envolvente: E.F. como hecho y práctica social } \\
\hline & \multicolumn{3}{|c|}{ Núcleos temáticos } \\
\hline & - Hombre y vida & $\begin{array}{l}\text { - Educación y } \\
\text { pedagogía }\end{array}$ & $\begin{array}{l}\text { - Experiencias } \\
\text { corporales y proceso } \\
\text { de desarrollo del } \\
\text { potencial humano }\end{array}$ \\
\hline \multicolumn{4}{|l|}{ PROFUNDIZACIÓN } \\
\hline \multirow[t]{3}{*}{$\begin{array}{l}\text { Nivel 1. construcción } \\
\text { disciplinar }\end{array}$} & \multicolumn{3}{|c|}{ Tema envolvente: E.F. Curricularizada } \\
\hline & \multicolumn{3}{|c|}{ Núcleos temáticos } \\
\hline & $\begin{array}{l}\text { - Hombre, cultura } \\
\text { y sociedad }\end{array}$ & $\begin{array}{l}\text { - Modelos } \\
\text { pedagógicos }\end{array}$ & $\begin{array}{l}\text { - Tecnica de } \\
\text { movimiento corporal }\end{array}$ \\
\hline \multirow[t]{2}{*}{ Nivel 2. Proyección } & \multicolumn{3}{|c|}{ Núcleos temáticos } \\
\hline & $\begin{array}{l}\text { Proyecto de } \\
\text { desarrollo } \\
\text { humano social y } \\
\text { cultural }\end{array}$ & $\begin{array}{l}\text { - Diseño de modelos } \\
\text { pedagógicos }\end{array}$ & $\begin{array}{l}\text { - Diseño } \\
\text { movimiento }\end{array}$ \\
\hline
\end{tabular}


La implementación de los Ciclos se hace con-creta a través del desarrollo de los campos de formación denominados: "Formación disciplinar específica y formación científica (tecnológica o artística) e Investigativa" (DCI) ; "Formación pedagógica y didáctica" (PD) y "Formación deontológica y en valores, campo en el cuál están incluidas la "Formación estética, recreativa y lingüística" (DVERL).

El Campo de Formación DCl, “...comprende un conjunto de temas problemas alrededor de los núcleos problemáticos, propios de una disciplina especifica o de los saberes interdisciplinarios y afines que cada proyecto curricular delimite..."

El Campo de Formación PD, “...es pera proporcionar al futuro educador oportunidades para elaborar y perfeccionar las competencias cognitivas, lingüísticas, investigativas y comunicativas que lo identifican como profesional de la educación..."

El Campo de Formación DVERL, “... constituye un espacio académico de praxis que busca la construcción de una conciencia de que todo saber es intersubjetivo y, por tanto, obedece a acuerdos y compromisos entre los miembros de una comunidad académica. Sus prácticas sociales se dan en el contexto más amplio de la cultura frente al cual se precisa desarrollar una conciencia profesional de servicio público y de construcción de valores..."

El desarrollo de los campos de formación se con-creta en los Espacios de Encuentro Académico, en los cuales se trabajaron los contenidos temáticos.

\section{INTENSIDAD HORARIA DE LOS CAMPOS DE FORMACIÓN}

\begin{tabular}{|c|c|c|c|}
\hline Campo de formación & Total horas/semana & Total horas/semestre & Total horas/carrera \\
\hline $\begin{array}{c}\mathrm{DCl} \\
80 \text { créditos * }\end{array}$ & $\begin{array}{c}20 \\
\text { Presenciales } 16 \\
\text { Trabajo individual } 4\end{array}$ & $\begin{array}{c}360 \\
\text { Presenciales } 288 \\
\text { Trabajo individual } 72\end{array}$ & $\begin{array}{c}3600 \\
\text { Presenciales } 2880 \\
\text { Trabajo individual } 720\end{array}$ \\
\hline $\begin{array}{c}\text { PD } \\
50 \text { Créditos }\end{array}$ & $\begin{array}{c}12,5 \\
\text { Presenciales } 10 \\
\text { Trabajo individual } 2,5\end{array}$ & $\begin{array}{c}225 \\
\text { Presenciales } 180 \\
\text { Trabajo individual } 45\end{array}$ & $\begin{array}{c}2250 \\
\text { Presenciales } 1800 \\
\text { Trabajo individual } 450\end{array}$ \\
\hline $\begin{array}{l}\text { DVERL } \\
30 \text { Créditos }\end{array}$ & $\begin{array}{c}7,5 \\
\text { Presenciales } 4 \\
\text { Trabajo individual 3,5 }\end{array}$ & $\begin{array}{c}135 \\
\text { Presenciales } 72 \\
\text { Trabajo individual } 63\end{array}$ & $\begin{array}{c}1350 \\
\text { Presenciales } 720 \\
\text { Trabajo individual } 630\end{array}$ \\
\hline
\end{tabular}

* 1 crédito $=45$ horas 
CICLOS

CAMPOS DE FORMACIÓN

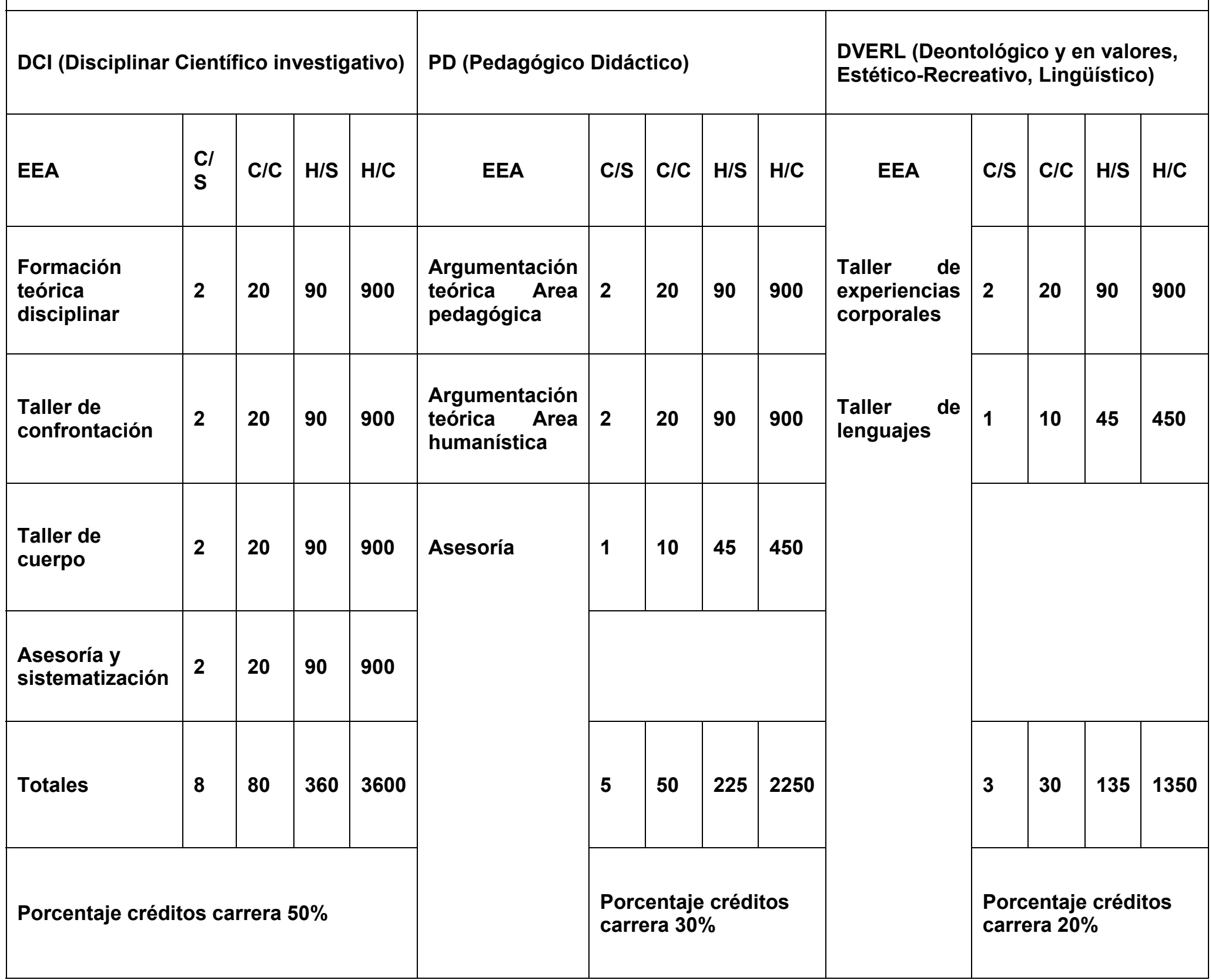

EEA Espacios de Encuentro Académico

C/S Créditos Semestre

C/C Créditos Carrera

H/S Horas Semestre

H/C Horas Carrera 


\section{CONTENIDOS TEMÁTICOS PARA LOS ESPACIOS \\ DE ENCUENTRO ACADÉMICO (EEA)}

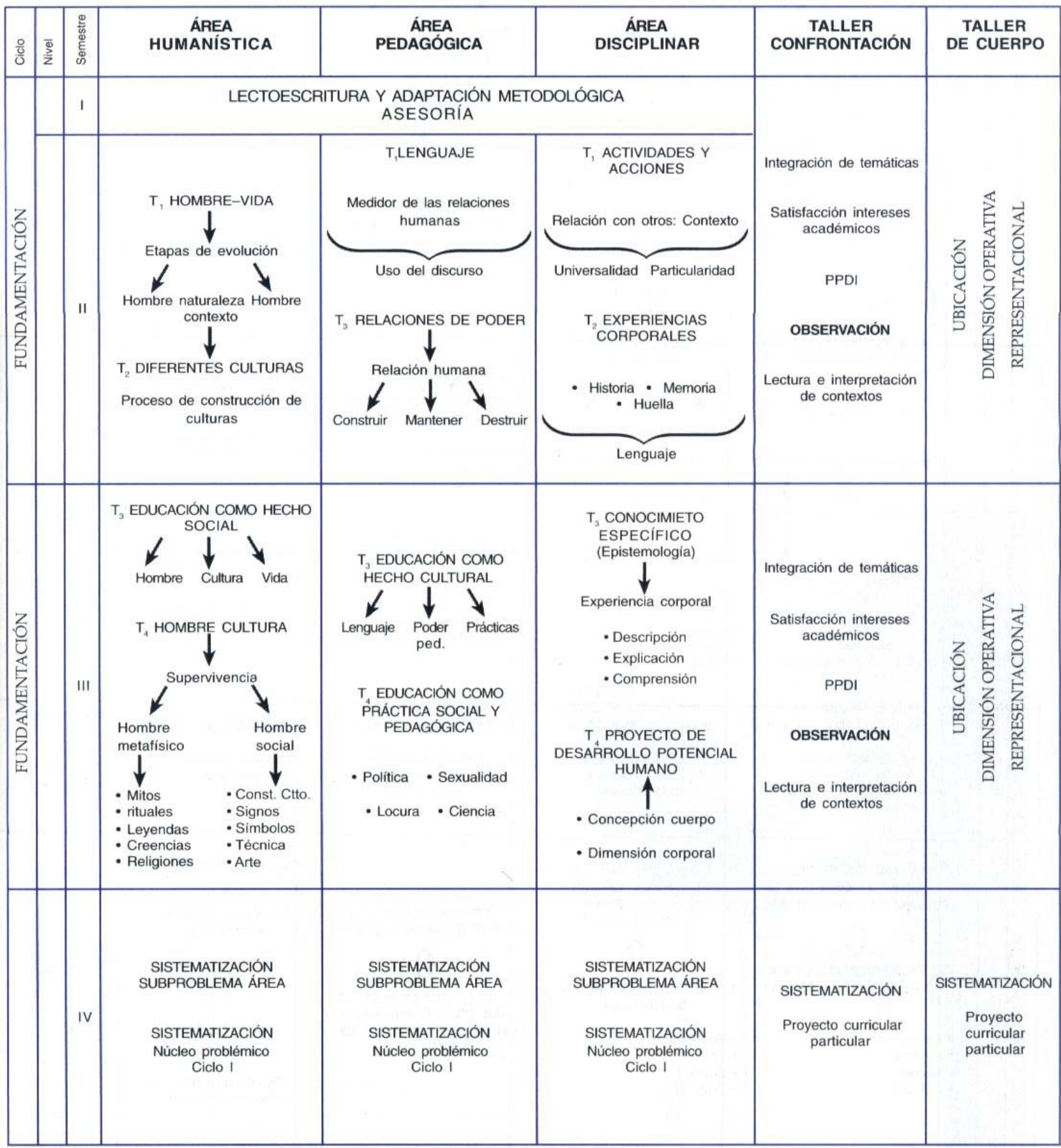




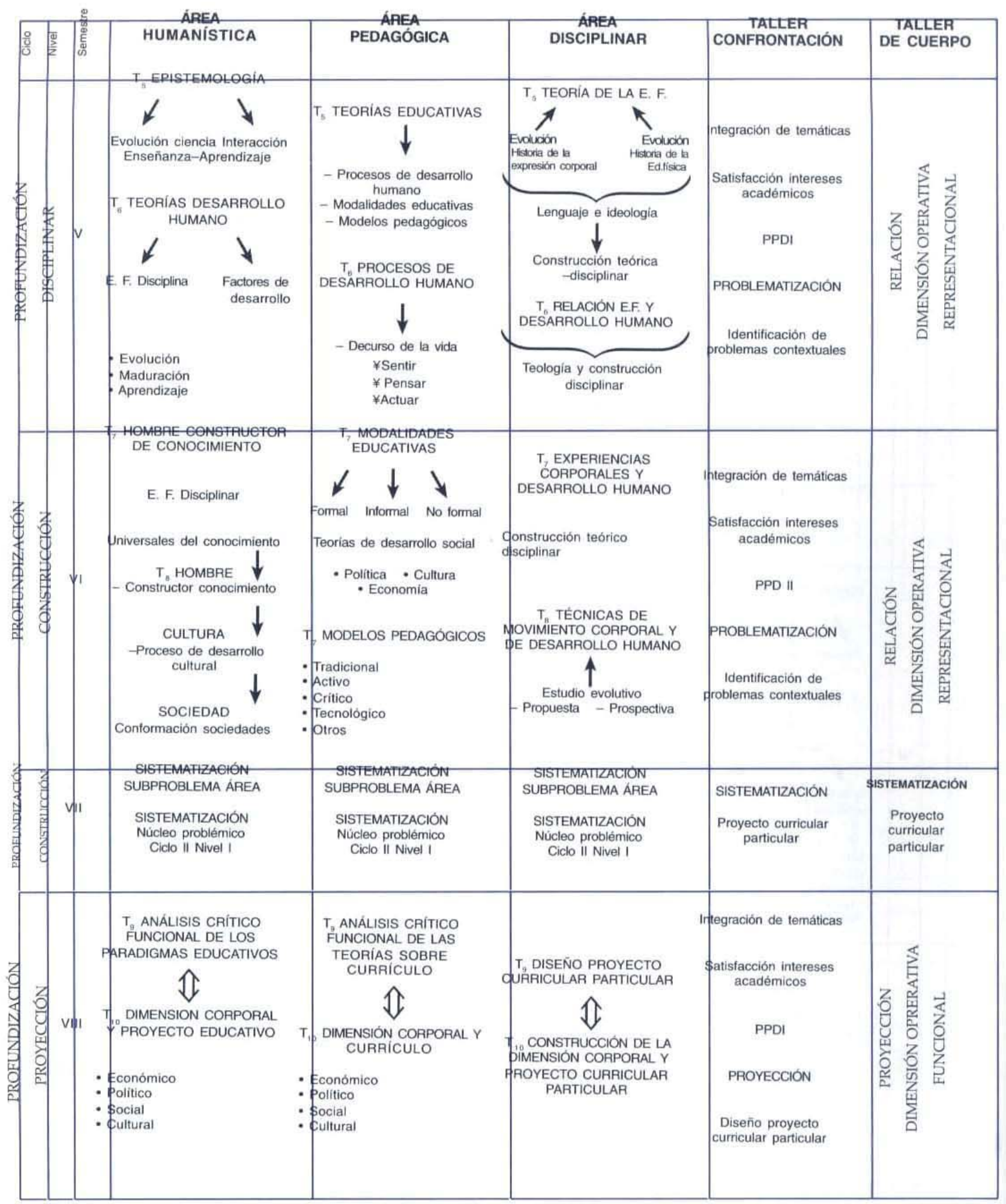




\begin{tabular}{|c|c|c|c|c|c|c|c|}
\hline$\frac{0}{0}$ & $\frac{\bar{\Phi}}{2}$ & 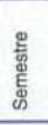 & $\begin{array}{c}\text { ÁREA } \\
\text { HUMANÍSTICA }\end{array}$ & $\begin{array}{c}\text { ÁREA } \\
\text { PEDAGÓGICA }\end{array}$ & $\begin{array}{c}\text { ÁREA } \\
\text { DISCIPLINAR }\end{array}$ & $\begin{array}{c}\text { TALLER } \\
\text { CONFRONTACIÓN }\end{array}$ & $\begin{array}{l}\text { TALLER } \\
\text { DE CUERPO }\end{array}$ \\
\hline 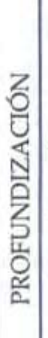 & 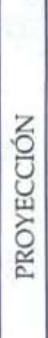 & IX & $\begin{array}{c}T_{11} \text { IMPLICACIONES ÉTICO } \\
\text { POLITICAS DE LOS } \\
\text { PROYECTOS EDUCATIVOS } \\
\Uparrow \\
\text { T } \\
T_{12} \text { IMPLICACIONES ÉTICO } \\
\text { POLITICAS DEL } \\
\text { PROYECTO CURRICULAR } \\
\text { PARTICULAR }\end{array}$ & 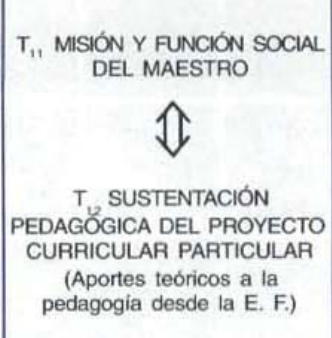 & 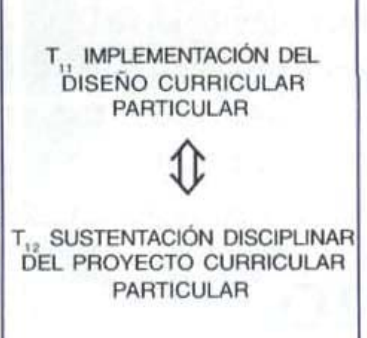 & $\begin{array}{l}\text { Integración de temáticas } \\
\text { Satisfacción intereses } \\
\text { académicos } \\
\text { PPD IV } \\
\text { PROYECCIÓN } \\
\begin{array}{c}\text { Implementación creativa } \\
\text { del proyecto curricular } \\
\text { particular }\end{array}\end{array}$ & 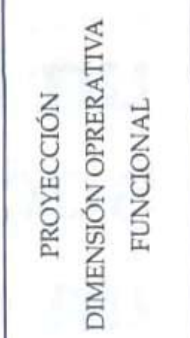 \\
\hline 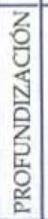 & 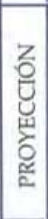 & $x$ & $\begin{array}{l}\text { SISTEMATIZACIÓN } \\
\text { PROYECTO CURRICULAR }\end{array}$ & $\begin{array}{l}\text { SISTEMATIZACIÓN } \\
\text { PROYECTO CURRICULAR }\end{array}$ & $\begin{array}{l}\text { SISTEMATIZACIÓN } \\
\text { PROYECTO CURRICULAR }\end{array}$ & $\begin{array}{l}\text { EVALUACIÓN DEL } \\
\text { PROYECTO } \\
\text { CURRICULAR } \\
\text { PARTICULAR }\end{array}$ & $\begin{array}{l}\text { EVALUACIÓN } \\
\text { DEL DISENO DE } \\
\text { MOVIMIENTO }\end{array}$ \\
\hline
\end{tabular}

$\Downarrow$ Relación de afectación.

$\downarrow$ Enunciados derivados.

\{ Enunciados de cohesión.

- Enunciados de pertenencia.

T Núcleo temático.

$\uparrow$ Enunciados de incidencia.

Æ Enunciados de co-afectación.

\section{Información complementaria}

Exigencias para la graduación: Haber cursado y aprobado los diez semestres del Plan de Estudio y sistematizar, socializar y sustentar el proyecto curricular particular.

Sobre el título: El tipo de modalidad y nivel, serán los que especifica la Ley 115, su escogencia para precisar el título, estará definida por el estudiante según el énfasis por el que opte al diseñar su proyecto curricular particular. 